than $2 \mathrm{mg}$. Nobody was given more than $9 \mathrm{mg}$. In total, $350 \mathrm{mg}$ of atropine was immediately necessary on the site of the attack, equivalent to 350 phials of $1 \mathrm{mg}$. In our simulation, the time for access and preparation of the antidote was about 10 minutes from the moment of the alert. The transfer and distribution time to the site was less than 15 minutes due to favourable road access, geographical factors and the short distance from the station to the storage facility.

Conclusions The pharmacist is responsible for immediate availability, accessibility and distribution of the antidotes to the site of emergency, and awareness of appropriate treatment.

No conflict of interest.

\section{GRP-186 THE QUALITY OF ORAL CHEMOTHERAPY PRESCRIBING IN ONCOHAEMATOLOGIAL OUTPATIENTS}

doi:10.1136/ejhpharm-2013-000276.186

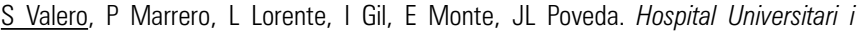
Politecnic La Fe, Hospital Pharmacy, Valencia, Spain

Background Nowadays, in our health area, most of the oral antineoplastic drugs prescribed to outpatients are dispensed in hospital pharmacy services. Patients receiving these kinds of drugs are susceptible to suffering adverse events (AE) due to medicines errors (MEs). Purpose To evaluate the quality of oral chemotherapy drug prescriptions (OCDPs) in oncohaematological outpatients.

Materials and Methods Descriptive prospective study. OCDPs for adult patients received in a pharmaceutical outpatient care unit were analysed for two months. The information necessary for OCDPs was established based on legal rules and international recommendations. We established that omitted or confused information in patient identification (identification number), weight, height and/or corporal surface (in drugs dosed depending on these parameters), diagnosis, treatment duration, dose and frequency of administration, presented serious risk based on possible consequences.

Results 291 prescriptions were analysed from 183 patients. 100\% of prescriptions had almost one omission, $78.7 \%$ of which showed serious errors of omitted or confused information related to the following items: patient identification $(0.7 \%)$, weight, height or corporal surface $(56.7 \%)$, diagnosis $(28.9 \%)$, treatment duration $(14.1 \%)$, dose $(5.8 \%)$ or frequency $(12.1 \%)$. Information omitted or confused about patient and treatment information included: age or birth date $(1.4 \%)$, allergies (omitted $56 \%$, unknown $38.8 \%$ ), morbidities $(59.5 \%)$, cycle number $(67 \%)$ and periodicity $(46.7 \%)$. Drug information omitted or confused included: drug name (generic $35.7 \%$, originator $61.5 \%$ or both $2.7 \%$ ), dose units $(10.7 \%)$, pharmaceutical form $(83.1 \%)$ or route of administration $(58.4 \%)$. Physician information omitted or confused included: name $(7.6 \%)$, signature $(1 \%)$ and collegiate number (1\%).

Conclusions Our results show a high rate of omitted and confused information in prescriptions in OCDP. Extreme attention during the validation process was required in order to prevent MEs and AEs. New tools, such as electronic prescription, pre-printed medical orders or educational programmes for prescribers, must be implemented in order to improve the quality of OCDP.

No conflict of interest.

\section{GRP-187 THE RATES AND TYPES OF PRESCRIBING ERRORS IN ELECTRONIC CHEMOTHERAPY PRESCRIPTIONS FOR AMBULATORY PATIENTS}

doi:10.1136/ejhpharm-2013-000276.187

'M Dobravc Verbic, ${ }^{2} \mathrm{~K}$ Kantilal, ${ }^{3} \mathrm{~N}$ Barber. ' UNIVERSITY MEDICAL CENTRE LJUBLJANA, Pharmacy, Ljubljana, Slovenia; 'Guy's and St Thomas' NHS Foundation Trust, Pharmacy, London, UK; ' UCL School of Pharmacy, Department of Practice and Policy, London, UK
Background Electronic prescribing (EP) systems have been recognised as successful in reducing chemotherapy prescribing errors. However, electronic prescriptions are unlikely to prevent all errors, and new types of errors may emerge.

Purpose To assess prescribing error rates and identify new error types and their causes with the implementation of a electronic prescribing system for ambulatory cancer patients at a London Cancer Centre.

Materials and Methods A service evaluation was conducted in two parts, covering two different strategies for interception of prescribing errors - prospectively by pharmacists during a 2 -week period, and retrospectively using data from the pharmacy EP telephone helpline service, over 41 weeks.

Results The overall rate of error-containing prescriptions was estimated to be $6 \%$.

In the prospective part, 32 errors were identified from 571 electronic chemotherapy prescriptions. Most commonly committed errors were chemotherapy drug dose adjustments (13; 41\%) and weight omissions (11; 34\%).

In the retrospective analysis, 95 of 141 errors (67\%) were 'e-selection errors', classified mainly as 'work-arounds' (26; 18\%), 'wrong commands' (35; 25\%), or 'wrong fields' (27; 19\%). 63 errors (45\%) were related to scheduling a chemotherapy or supportive drug or regimen.

Electronic system-related causes of prescribing errors were recognised in 4 of 32 cases (13\%) in the prospective part, and in 89 of 141 cases $(63 \%)$ in the retrospective part. It was estimated that with implementation of technical solutions and additional prescriber training, $85 \%$ of these errors could be prevented in the future.

Conclusions The estimated rate of chemotherapy prescribing errors was $6 \%$. A number of different errors, specific for electronic prescribing, were identified, with a thorough explanation of how various errors may have occurred. Future larger scale studies are needed to confirm prescribing error rates, and to possibly identify other, previously unrecognised, types of chemotherapy prescribing errors.

No conflict of interest.

\section{GRP-188 UNDER-REPORTING OF ADVERSE DRUG REACTIONS IN THE HOSPITAL SETTING: AN ESTIMATE BASED ON THE ANALYSIS OF HOSPITAL DISCHARGE RECORDS}

doi:10.1136/ejhpharm-2013-000276.188

'MC Leo, 'E Cini, ${ }^{2} \mathrm{~A}$ Pugi, 'R Banfi. 'Careggi University Hospital, Pharmacy, Florence, Italy; ${ }^{2}$ University of Florence, Centre of Pharmacovigilance Department of Pharmacology, Florence, Italy

Background In the post-marketing setting, spontaneous reporting is an important tool for the surveillance of Adverse Drug Reactions (ADRs). However, underreporting is a major limitation of a pharmacovigilance system. Several studies showed that ADRs may cause hospitalisation resulting in an increase in hospital stays and costs.

Purpose To gather information on the extent and frequency of ADRs at Careggi University Hospital, and to identify unreported ADRs to the Pharmacovigilance Office, using the hospital discharge records.

Materials and Methods We analysed the hospital discharge records from January 2011 to June 2012. In particular, we considered those records with a Drug Related Group (DRG) classification related to allergic reactions, poisoning and toxic effects of drugs (DRGs from 447 to 451). We included in our analysis records referring to poisoning, according to the new pharmacovigilance legislation in force from July 2012. Our research gave us information about the number of suspected reactions, but it didn't provide specific information on the patients and the seriousness of the reaction. 